\title{
Does cardiovascular therapy affect the onset and recurrence of preretinal and vitreous haemorrhage in diabetic eye disease?
}

S Banerjee ${ }^{1,2}$, AKO Denniston ${ }^{1,2}$, JM Gibson ${ }^{1,2}$ and PM Dodson ${ }^{2}$

\begin{abstract}
Aims To review the role of cardiovascular disease and therapy in the onset and recurrence of preretinal/vitreous haemorrhage in diabetic patients.
\end{abstract}

Methods Retrospective case note analysis of diabetic patients with vitreous haemorrhage from the Diabetic Eye Clinic at Birmingham Heartlands Hospital.

Results In total, 54 patients (mean age 57.1, 37 males, 20 type I vs 34 type II diabetic patients) were included. The mean (SD) duration of diagnosed diabetes at first vitreous haemorrhage was significantly longer, 21.9 (7.6) years for type I and 14.8 (9.3) years for type II diabetic patients $(P<0.01$, unpaired $t$-test, two-tailed).

Aspirin administration was not associated with a significantly later onset of vitreous haemorrhage. Four episodes were associated with ACE-inhibitor cough. There was a trend towards HMGCoA reductase inhibitor (statin) use being associated with a delayed onset of vitreous haemorrhage: 21.4 years until vitreous haemorrhage (treatment group) vs $\mathbf{1 6 . 2}$ years (nontreatment group) $(P=0.09$, two-tailed, unpaired $t$-test, not statistically significant).

During follow-up 56 recurrences occurred, making a total of $\mathbf{1 1 0}$ episodes of vitreous haemorrhage in 79 eyes of 54 patients. The mean (range) follow-up post haemorrhage was 1067 (77-3842) days, with an average of 1.02 recurrences. Age, gender, diabetes type (I or II) or control, presence of hypertension or hypercholesterolaemia, and macrovascular complications were not associated with a significant effect on the 1-year recurrence rate.
Aspirin (and other antiplatelet or anticoagulant agents) and ACE- inhibitors appeared to neither increase nor decrease the 1-year recurrence rate. However, statin use was significantly associated with a reduction in recurrence (Fisher exact $\boldsymbol{P}<0.05$; two-tailed) with an odds ratio (95\% CI) of 0.25 (0.1-0.95). Conclusion In this retrospective analysis, the onset of preretinal/vitreous haemorrhage was not found to be accelerated by gender, hypertension, hypercholesterolaemia, evidence of macrovascular disease, or HbA1c. Neither aspirin nor ACE-inhibitor administration accelerated the onset or recurrence of first vitreous haemorrhage. Statins may have a protective role, both delaying and reducing the recurrence of haemorrhage.

Eye (2004) 18, 821-825. doi:10.1038/sj.eye.6701338

Published online 20 February 2004

Keywords: vitreous haemorrhage; diabetic retinopathy; aspirin; statin

\section{Introduction}

Diabetes mellitus is a major cause of visual morbidity worldwide. Although the incidence and burden of the disease is increasing, the recognition of modifiable risk factors is giving opportunities for improving visual outcome in these patients. The major modifiable risk factors comprise glycaemic control (demonstrated by DCCT for type I patients and by UKPDS for type II disease $)^{1-4}$ and hypertension. ${ }^{5}$ Several subsidiary factors have been identified more
${ }^{1}$ Birmingham and Midland Eye Centre Birmingham, UK

${ }^{2}$ Birmingham Heartlands and Solihull Trust Birmingham, UK

Correspondence: PM Dodson Birmingham Heartlands and Solihull

NHS Trust

Bordesley, Green East

Birmingham, West Midlands B9 5SS, UK

Tel: + 441214242000

Fax: +441214245462

E-mail: Paul.Dodson@

heartsol.wmids.nhs.uk

Received: 22 December 2002

Accepted: 22 July 2003

Published online: 20

February 2004

Conflict of interest: None Presented in part at the International Congress of Ophthalmology, Sydney, April 2002 and the Annual Congress of Royal College of Ophthalmologists, Manchester, May 2002 
recently: hyperlipidaemia appears to have a role in the progression of maculopathy for type II patients; ${ }^{6,7}$ similarly, nephropathy (probably through its effects on lipid profiles and blood pressure) is implicated in the progression of maculopathy. ${ }^{6}$

It might thus seem that control of well-established risk factors for macrovascular disease (glycaemia,

hypertension, hyperlipidaemia, smoking) would result in benefits in retinopathy also. Certainly, hypertension has been effectively combated with a range of well-tolerated agents that are associated with reduction in the development and progression of retinopathy (in type II disease) ${ }^{4,5}$ there remain concerns however that the use of ACE inhibitors with their frequent attendant cough may precipitate vitreous haemorrhage. Also, while HMGCoA reductase inhibitors ('statins') have impressive benefits in reducing cholesterol with associated reduction in myocardial infarct, stroke, and cardiovascular mortality, their effect on retinopathy is not known. Another agent that is acclaimed for its role against macrovascular disease but has no proven benefit in retinopathy is aspirin. ${ }^{8-10}$ Indeed, considerable debate has raged over whether it may increase the risk and severity of vitreous haemorrhage. ${ }^{11-13}$ The importance of this issue has been highlighted by the advice from Diabetes UK and the American Diabetic Association that all patients with diabetic retinopathy who are over 30 years of age should be taking aspirin. Lastly, while smoking is prohibited on account of its profound effects on macrovascular disease, it is actually associated with reduced development and progression of retinopathy in type II diabetic patients. ${ }^{5}$ The purpose of this retrospective study was to assess a series of patients in terms of cardiovascular risk and their risk modification (antihypertensives, cholesterollowering therapies, aspirin) with specific emphasis on aspirin therapy. Outcomes considered were time to first preretinal/vitreous haemorrhage and recurrence of preretinal/vitreous haemorrhage; this end point was chosen both on account of the specific concerns regarding aspirin (and other antiplatelet/anticoagulant agents) and as a clearly defined marker of advanced diabetic eye disease.

\section{Materials and methods}

\section{Study design}

Retrospective analysis of case notes of all patients with recent and active preretinal/vitreous haemorrhage attending the Diabetic Retinopathy Clinic at Birmingham Heartlands and Solihull Hospital. This has an urban catchment area. Patients attending the clinic between January 2001 and December 2001 were included in this study.

\section{Proforma}

The following data were collected: demographic (age, sex, race, eye); diabetic history (type I or II, duration, treatment); cardiovascular risk (history and duration of hypertension, history and duration of hypercholesterolaemia); hypertension and hypercholesterolaemia were defined by our diabetologists as requiring drug treatment according to primary or secondary prevention targets as recommended by the Joint British Guidelines. ${ }^{14}$ In those patients who had had a macrovascular disease event (24\% in this series), statin therapy was indicated if serum cholesterol was $>4.8 \mathrm{mmol} / \mathrm{l}$. The remaining patients had cardiovascular risk management according to primary prevention targets consisting of antihypertensive treatment if blood pressure was $>140$ / 85 and statin and aspirin therapy if their 10 year coronary heart disease (CHD) risk was $>15 \%$ calculated by the Framingham equation: ${ }^{15}$ cardiovascular comorbidity (history of myocardial infarct, stroke, or peripheral vascular disease); cardiovascular therapy (use of antihypertensives, lipid-lowering agents, antiplatelet agents, or anticoagulants); ophthalmic history (timing of vitreous/preretinal haemorrhage, associated events including trauma or surgery, most recent assessment of retinopathy, presence and extent of previous laser treatment, previous operations).

\section{Results}

The details of 54 patients with the first onset of vitreous haemorrhage were identified (Table 1). Of these, 20 patients had type I and 34 patients had type II disease. The mean age was 57.1 years (higher for type II disease than type I disease as expected). There was a preponderance of men, with 37 male and 17 female subjects. The mean (SD) duration of diagnosed diabetes at first vitreous haemorrhage was 21.9 (7.6) years for type I and 14.8 (9.3) years for type II diabetic patients. This represents a significant difference $(P<0.01$, unpaired $t$-test, two-tailed).

In $91 \%$ of first episodes of preretinal/vitreous haemorrhage, proliferative retinopathy was present; in $7 \%$ of cases acute posterior vitreous detachment was thought to be causative or contributory (Table 2).

Hypertension was present in $68 \%$, hypercholesterolaemia in $33 \%$, and both factors in $25 \%$ of patients; $24 \%$ had already suffered a cardiovascular event such as myocardial infarction or stroke.

Statins were being prescribed to 14 patients $(26 \%)$, with a mean (SD) duration of treatment of 6.9 (4.0) years. There was a trend towards statin use being associated with a delayed onset of vitreous haemorrhage: 21.4 years 
Table 1 Demographic and cardiovascular history of first episodes of preretinal/vitreous haemorrhage

\begin{tabular}{|c|c|c|c|c|}
\hline & All & Type I & Type II & $\begin{array}{l}\text { Significant } \\
\text { difference }\end{array}$ \\
\hline \multicolumn{5}{|l|}{ Demographics } \\
\hline Number & 54 & 20 & 34 & \\
\hline Mean age & 57.1 years & 44.2 years & 65.1 years & \\
\hline Male: female & 2.2 & 1.2 & 3.3 & \\
\hline \multicolumn{5}{|l|}{ Cardiovascular history } \\
\hline Hypertensive & $69 \%$ & $70 \%$ & $67 \%$ & NS \\
\hline Mean (SD) duration of hypertension & $6.1(5.1)$ years & $4.3(2.8)$ years & $7.3(6.0)$ years & NS \\
\hline Hypercholesterolaemia & $33 \%$ & $25 \%$ & $38 \%$ & NS \\
\hline Mean (SD) duration of hypercholesterolaemia & $5.0(3.0)$ years & $5.3(4.0)$ years & 4.9 (2.9) years & NS \\
\hline Hypertensive and hypercholesterolaemic (included above) & $26 \%$ & $20 \%$ & $29 \%$ & NS \\
\hline \multicolumn{5}{|l|}{ Level of $\mathrm{HbA1c}$} \\
\hline Mean (SD) HbA1c at first episode & $7.9(2.0)$ & $8.4(1.7)$ & $7.6(2.2)$ & NS \\
\hline
\end{tabular}

$\mathrm{NS}=$ no significant difference. Linear regression analysis also shows no correlation between $\mathrm{HbA1c}$ and onset of haemorrhage.

Table 2 Cause of first episode of vitreous haemorrhage according to stage of retinopathy

\begin{tabular}{lcc}
\hline & $\begin{array}{l}\text { Proliferative } \\
\text { diabetic retinopathy } \\
(\mathrm{n}=49)\end{array}$ & $\begin{array}{l}\text { Non proliferative } \\
\text { diabetic retinopathy } \\
(\mathrm{n}=5)\end{array}$ \\
\hline $\begin{array}{l}\text { Neovascularisation } \\
\text { Posterior vitreous }\end{array}$ & 49 & 0 \\
detachment & 3 & 1 \\
Cough/sneeze & 4 & 0 \\
Uncertain cause & 0 & 4 \\
\hline
\end{tabular}

$\mathrm{NB}$, Some cases were multifactorial.

until vitreous haemorrhage (treatment group) vs 16.2 years (nontreatment group) $(P=0.09$, two-tailed, unpaired $t$-test). However, this was not statistically significant (see Table 3).

Aspirin was being taken by $23(43 \%)$ patients, with a mean (SD) duration of 3.8 (2.5) years. One patient was also taking warfarin. Interestingly, no patients were taking other antiplatelet agents such as clopidogrel or persantin. Aspirin usage was not found to be associated with a significantly later onset of vitreous haemorrhage. ACE inhibitors were being administered to 31 (57\%) patients. Although four episodes were associated with ACE-inhibitor cough, overall there was no significant association between ACE inhibitors and earlier vitreous haemorrhage.

In terms of 1-year recurrence, aspirin (and other antiplatelet or anticoagulant agents) and ACE inhibitors appeared to neither increase nor decrease that 1-year recurrence rate. However, statin use was significantly associated with a reduction in recurrence (Fisher exact $P<0.05$; two-tailed) with an odds ratio $(95 \% \mathrm{CI})$ of 0.25 (0.1-0.95).
Table 3 Effect of aspirin and statins

\begin{tabular}{ll}
\hline $\begin{array}{l}\text { Time to first } \\
\text { haemorrhage }\end{array}$ & All patients \\
On aspirin & $17.9(10.3)$ years \\
Not on aspirin & $17.1(8.7)$ years \\
Significant difference & NS \\
& $21.4(8.5)$ years \\
On statin & $16.2(9.3)$ years \\
Not on statin & NS, P=0.09 \\
Significant difference & All patients \\
& $23 \%$ \\
1-year recurrence rate & $15 \%$ \\
On aspirin & $\mathrm{NS}$ \\
Not on aspirin & $10 \%$ \\
Significant difference & $31 \%$ \\
On statin & $P<0.05$ \\
Not on statin & \\
Significant difference &
\end{tabular}

\section{Discussion}

Vitreous haemorrhage is a significant complication of diabetic eye disease, which usually results in a deterioration in visual acuity, and with unpredictable recovery. This retrospective study gives a useful profile of this group of vulnerable patients. In addition to the presence of diabetes (usually in excess of a 15 year duration since diagnosis), other cardiovascular risk factors are usually present. Indeed, $69 \%$ were hypertensive, $33 \%$ hypercholesterolaemic, and $26 \%$ had both risk factors. It is perhaps not surprising then that $24 \%$ of these patients have had a myocardial infarct or stroke by the time of first preretinal/vitreous haemorrhage.

Hypertensive control reduces development and progression or retinopathy. ${ }^{3,4}$ Concern has been 
expressed that the ACE inhibitor cough might result in vitreous haemorrhage in vulnerable eyes. This study found that four episodes of first vitreous haemorrhage were associated with such a cough. However, the timing of the first vitreous haemorrhage was not significantly earlier in the ACE inhibitor treatment group when compared to the rest of the cohort. Larger studies may demonstrate whether there is a group of patients (for example, with active proliferative diabetic retinopathy) in whom ACE inhibitors should be replaced by another agent such as angiotensin II receptor antagonists. A further issue is the rate of undiagnosed or undertreated hypertension in this group. One recent study suggested that in up to $72 \%$ of diabetic patients, their blood pressure is poorly controlled and that $23 \%$ had undiagnosed hypertension. ${ }^{16}$

The decreasing threshold for treatment of hypercholesterolaemia means that many diabetic patients should now be taking statins. However, our data suggest that there was underprescribing of statins in our patient cohort as, according to the Joint British Guidelines, ${ }^{14}$ in excess of $80 \%$ of our patients would have been eligible based on CHD risk $>15 \%$ or previous macrovascular disease event. An unexpected result of this study was that these agents appeared to have a protective role in delaying the onset of first vitreous haemorrhage. The mechanism of this is uncertain. Statins have been shown to stabilise atherosclerotic plaques within vessels, and might indirectly contribute to the strength of the vessel wall. This would then solely influence the risk of haemorrhage in at-risk eyes. Perhaps more likely (and of more interest) is the possibility that delayed vitreous haemorrhage is reflecting a deceleration in the progression of retinopathy. Lastly, treatment with statins may actually be a surrogate marker for some more important factor, such as better general medical care. Thus, any potential benefit of statins in this context should be confirmed prospectively in a randomised controlled trial.

Given the high cardiovascular risk and of demonstrable macrovascular disease, it is perhaps surprising that only $43 \%$ of patients were taking aspirin. The Early Treatment of Diabetic Retinopathy Study (ETDRS) Report No. 20 found that the use of aspirin at a dose of $650 \mathrm{mg} /$ day did not increase the occurrence of vitreous/preretinal haemorrhage. Other authors have suggested that the use of aspirin, antiplatelet, and anticoagulant therapy in the presence of proliferative diabetic retinopathy is contraindicated due to the increased risk of vitreous and preretinal haemorrhage. Our limited data are in line with the ETDRS in suggesting that the widespread use of aspirin in diabetic patients with retinopathy is safe.
For patients with diabetic retinopathy and vitreous haemorrhage, there is a significant risk of recurrence with a 1-year repeat haemorrhage rate of $24 \%$. However, such recurrences were only seen in patients with proliferative diabetic retinopathy; haemorrhage in the context of BDR or PPDR was a rare event, generally associated with PVD or trauma and therefore intrinsically less likely to recur.

Interestingly, statin use appeared to be beneficial in reducing haemorrhage recurrence (Fisher exact $P<0.05$; two-tailed) with an odds ratio (95\% CI) of 0.25 (0.1-0.95). In contrast, aspirin (and other antiplatelet agents) and ACE inhibitors were not associated with a change in haemorrhage recurrence rate.

Our study suggests that the prescription of aspirin, ACE inhibitors and statins to diabetic patients does not increase the risk of preretinal and vitreous haemorrhage, and that statins may indeed have a beneficial effect. The study was not sufficiently powered to demonstrate a possible association between statins and time to first haemorrhage, as the aim of the study was to assess aspirin and vitreous haemorrhage. The major limitations of this study are its retrospective nature and the lack of control group. However, further investigation is underway with two large prospective randomised controlled trials, ASPEN and the CARDS study, due to report later in 2004. ${ }^{7,17,18}$ The CARDS study will support more widespread statin prescription in type II diabetic patients, and if the conclusions from this study are correct we may in turn see a delay or reduction in the episodes of preretinal or vitreous haemorrhage in diabetic patients.

\section{References}

1 DCCT Research group. The effect of intensive treatment of diabetes on the development and progression of long-term complications in insulin dependent diabetes mellitus. N Engl J Med 1993; 329: 977-986.

2 UK Prospective Diabetes Study (UKPDS) Group. Intensive blood-glucose control with sulphonylureas or insulin compared with conventional treatment and risk of complications in patients with type 2 diabetes (UKPDS 33). Lancet 1998; 352(9131): 837-853.

3 UK Prospective Diabetes Study (UKPDS) Group. Effect of intensive blood-glucose control with metformin on complications in overweight patients with type 2 diabetes (UKPDS 34). Lancet 1998; 352(9131): 854-865.

4 Stratton IM, Kohner EM, Aldington SJ, Holman RR, Manley SE, Matthews DR. UKPDS50: risk factors for incidence and progression of retinopathy in Type II diabetes over 6 years from diagnosis. Diabetologia 2001; 44(2): 156-163.

5 UK Prospective Diabetes Study Group. UKPDS38: tight blood pressure control and risk of macrovascular and microvascular complications in type II diabetes. BMJ 1998; 317: 703-713. 
6 Aiello LP, Cahill MT, Wong JS. Systemic considerations in the management of diabetic retinopathy. Am J Ophthalmol 2001; 132(5): 760-766.

7 Chowdhury TA, Hopkins D, Dodson PM, Vafidis GC. The role of serum lipids in exudative diabetic maculopathy: is there a place for lipid lowering therapy? Eye 2002; 16: 689-693.

8 Antiplatelet Trialists' Collaboration. Colloborative overview of randomised trials of antiplatelet therapy, I. Prevention of death, myocardial infarction and stroke by prolonged antiplatelet therapy in various categories of patients. BMJ 1994; 308: 81-106.

9 Cleland JGF. Preventing atherosclerotic events with aspirin. BMJ 2002; 324: 103-105.

10 Antiplatelet Trailists' Collaboration. Collaborative metaanalysis of randomised trials of antiplatelet therapy for prevention of death, myocardial infarction and stroke in high risk patients. BMJ 2002; 324: 71-86.

11 Klein R, Marino EK, Kuller LH, Polak JF, Tracy RP, Gottdiener JS et al. The relation of atherosclerotic cardiovascular disease to retinopathy in people with diabetes in the Cardiovascular Health Study. Br J Ophthalmol 2002; 86: 84-90.

12 Chew EY, Klein ML, Murphy RP, Remaley NA, Ferris III FL. Effects of aspirin on vitreous/preretinal hemorrhage in patients with diabetes mellitus Early Treatment Diabetic Retinopathy Study Report No. Arch Ophthalmol 1995; 113: 52-55.

13 Kern TS, Engerman RL. Pharmacological inhibition of diabetic retinopathy; aminoguanidine and aspirin. Diabetes 2001; 50(7): 1636-1642.

14 Wood DA, Durrington P, McInnes G, Poulter N, Rees A, Wray R. Joint British recommendations on prevention of coronary heart disease in clinical practice. Heart 1998; 80 (Suppl 2): S1-S29.

15 Anderson KM, Odell PM, Wilson PW, Kammel WB. Cardiovascular disease risk profiles. Am Heart J 1991; 121: 293-298.

16 Al-Husainy S, Farmer J, Gibson JM, Dodson PM. Measurement of blood pressure in patients with diabetic retinopathy. Eye 2004 (in press).

17 Colhoun HM, Thomason MJ, Mackness MI, Maton SM, Betteridge DJ, Durrington PN et al. Collaborative AtoRvastatin Diabetes Study (CARDS). Design of the Collaborative AtoVastatin Diabetes Study (CARDS) in patients with type 2 diabetes. Diabet Med 2002; 19(3): 201-211.

18 Donaldson M, Dodson PM. Medical treatment of diabetic retinopathy. Eye 2003; 17: 550-562. 